\title{
Improving perioperative care for adolescent idiopathic scoliosis patients: the impact of a multidisciplinary care approach
}

REVIEW

\author{
Timothy C Borden \\ Laura L Bellaire \\ Nicholas D Fletcher \\ Department of Orthopaedic Surgery, \\ Emory University, Atlanta, GA, USA
}

\author{
This article was published in the following Dove Press journal: \\ Journal of Multidisciplinary Healthcare \\ 14 September 2016 \\ Number of times this article has been viewed
}

Correspondence: Nicholas D Fletcher Emory Orthopaedics and Spine Center, 59 Executive Park South - Ste 2000, Atlanta, GA 30329, USA

Tel +l 4047783350

Email nicholas.d.fletcher@emory.edu

\begin{abstract}
The complex nature of the surgical treatment of adolescent idiopathic scoliosis (AIS) requires a wide variety of health care providers. A well-coordinated, multidisciplinary team approach to the care of these patients is essential for providing high-quality care. This review offers an up-to-date overview of the numerous interventions and safety measures for improving outcomes after AIS surgery throughout the perioperative phases of care. Reducing the risk of potentially devastating and costly complications after AIS surgery is the responsibility of every single member of the health care team. Specifically, this review will focus on the perioperative measures for preventing surgical site infections, reducing the risk of neurologic injury, minimizing surgical blood loss, and preventing postoperative complications. Also, the review will highlight the postoperative protocols that emphasize early mobilization and accelerated discharge. Keywords: adolescent idiopathic scoliosis, multidisciplinary health care, outcomes, guidelines, complications
\end{abstract}

\section{Introduction}

Adolescent idiopathic scoliosis (AIS) is a lateral and rotational deformity of the spine defined by a radiographic Cobb angle of $>10^{\circ}$ affecting otherwise healthy children around the age of puberty. An estimated 1\%-3\% of children between the ages of 10 and 16 are affected by AIS with a 7:1 female to male ratio. ${ }^{1,2}$ While the vast majority of these children will not require any intervention for their spinal curvature, $\sim 0.26 \%$ of children with AIS will be treated with either bracing or surgery. ${ }^{2}$

In the US, over 4,500 surgeries were performed for the treatment of AIS in $2000 .^{3}$ Data compiled from the Scoliosis Research Society Morbidity and Mortality Committee estimate the complication rate for AIS surgery to be $5.7 \%$. The most common complications for posterior instrumentation and fusion included wound infection $(1.35 \%)$, pulmonary complications excluding pulmonary embolism $(0.95 \%)$, neurologic injury $(0.32 \%)$, and other medical complications. ${ }^{4}$

The complex nature of the surgical treatment of AIS requires a wide variety of different health care providers. During the perioperative period, the average pediatric spine patient will interact with many providers including preoperative nurses, anesthetist, anesthesiologists, scrub technicians, circulating nurses, spine surgeons, neurologists and neuromonitoring technicians, cell-saver and radiology technicians, intensive care/floor nurses, physical and occupational therapists, and hospitalists. While each provider may have a different sphere of influence in patient care (eg, preoperative, intraoperative, postoperative), safe and effective surgical treatment of AIS is the primary goal of every provider. 
Multidisciplinary teams that focus on establishing and implementing surgical site infection (SSI) prevention protocols have successfully reduced infection in pediatric spine patients. ${ }^{5}$ Expanding the scope of these teams to reduce all known complications for the entire perioperative period may have the same effect. The purpose of this paper is to outline the evidence-based interventions involved in safely caring for the AIS surgical patient.

\section{Reducing the risk of infection}

SSI can be a devastating complication for pediatric patients undergoing spinal surgery for AIS. Perioperative infection is the most common acute complication in AIS surgery with an incidence ranging between $0.5 \%$ and $6.7 \% .^{6}$ Infections after orthopedic surgery can negatively affect patient outcomes, result in greater physical limitations, and significantly reduce the quality of life. In addition to the impact on the patient, SSI prolongs hospital stay, results in more rehospitalizations, and increases direct health care costs. ${ }^{7}$ Postoperative infections are estimated to increase costs by $300 \%$ in orthopedic patients in general, with costs ranging from $\$ 26,977$ to $\$ 961,722$ (mean $\$ 155,000$ ) for spine implant-related infections. ${ }^{5,7,8}$

Prevention of infection is the responsibility of the entire health care team. Standardized protocols have been adopted in many centers to reduce the risk of SSI. Ballard et al reported a $50 \%$ relative risk reduction in pediatric spine surgery infections after the implementation of a multidisciplinary task force for SSI prevention. ${ }^{5}$ Their team consisted of delegates from orthopedic surgery, infectious disease, epidemiology, pharmacy, anesthesia, quality improvement, and operative staff who met monthly to update their protocols and track their effectiveness. ${ }^{5}$

Preadmission baths with chlorohexidine reduce the concentration of cutaneous bacteria. ${ }^{9}$ While there have been no studies in pediatric spine patients, preoperative chlorohexidine bathing prior to arrival at the hospital has been shown to be effective in reducing SSI in knee and hip arthroplasty. ${ }^{10,11}$ In the neuromuscular pediatric population, optimization of preoperative nutrition also lowers SSI. Jevsevar and Karlin found that maintaining serum albumin $>35 \mathrm{~g} / \mathrm{L}$ and total blood lymphocyte count $>1.5 \mathrm{~g} / \mathrm{L}$ resulted in a significant decrease in SSI and the length of hospital stay. ${ }^{12}$ Obesity has also been identified as a potential, modifiable SSI risk factor. ${ }^{13}$ In a retrospective case-control study, Linam et al found that a body mass index of greater than the 95 th percentile correlated with an increase in postoperative SSI and readmission. ${ }^{14}$ Also, providing patients with preoperative informational handouts on SSI prevention has been recommended as a low-cost, low-risk infection control method. ${ }^{15}$

Appropriate perioperative antimicrobial prophylaxis is a fundamental intervention that has greatly reduced SSI in pediatric spine deformity surgery. ${ }^{16}$ Coagulase-negative Staphylococcus aureus remains the most common organism responsible for SSI in AIS surgery. However, polymicrobial flora and gram-negative bacteria have also been linked to SSI in the AIS population. ${ }^{17}$ In two retrospective reviews of over 1,400 pediatric spine deformity surgeries which included both AIS and neuromuscular scoliosis patients, gram-negative organisms accounted for over $50 \%$ of SSI, with a growing incidence of infection from Pseudomonas aeruginosa. ${ }^{13,14}$ First-generation cephalosporins, namely, cefazolin, are the recommended antimicrobial prophylaxis for all spine patients. ${ }^{15}$ The use of clindamycin as the sole agent for perioperative antibiosis was an independent risk factor for SSI after pediatric scoliosis surgery. ${ }^{14}$ Some centers have shown a decrease in SSI after spine surgery for AIS with the use of routine ceftazidime and vancomycin instead of cefazolin. ${ }^{17}$ Current consensus-driven best practice guidelines for high-risk pediatric spine patients recommend routine perioperative intravenous (IV) cefazolin use, addition of gram-negative coverage, and adherence to timing, dosing, redosing, and cessation regimens. ${ }^{15}$ While these guidelines are directed toward high-risk patients, the infection control principles are based on the best available evidence and should be applied to the AIS patient.

Appropriate delivery of antibiotic prophylaxis is the responsibility of the entire operative team. Optimal dosing and timing are essential for an effective SSI prevention; cefazolin should be given within 30 minutes of incision at a dose $20 \mathrm{mg} / \mathrm{kg}$ (maximum 2,000 mg) and vancomycin should be given within 150 minutes of incision at a dose of $10 \mathrm{mg} /$ $\mathrm{kg}$ (maximum 1,000 mg) ${ }^{18}$ Labbe et al demonstrated that appropriate dosing and timing reduced SSI in pediatric spine patients with an odds ratio of 5.5. ${ }^{18}$ Dosing and timing of IV antibiotic prophylaxis should be included in the "time-out" protocol as it has been shown to be a cheap and effective way to ensure appropriate treatment. ${ }^{19}$ In addition to IV antibiotic prophylaxis, several studies have demonstrated the effectiveness and safety of routine use of vancomycin powder in the bone graft and/or the surgical site. ${ }^{20-22}$ Sweet et al retrospectively studied the use of $2 \mathrm{~g}$ of vancomycin powder in 911 of 1,732 AIS spine surgeries and found a reduction in deep infection rate from $2.6 \%$ to $0.2 \%{ }^{21}$

Increased operating room (OR) traffic, including frequent opening and closing of OR doors, disrupts the laminar airflow, increases the number of microbes surrounding the surgical 
field, and contributes to increased risk of infection. ${ }^{23}$ Panahi et al found that there was an average of 60 door openings during a primary total joint replacement lasting 92 minutes and an average of 135 openings during revision cases. ${ }^{23}$ In addition to limiting OR access, Vitale et al recommended several perioperative measures to prevent SSI in their 2013 Best Practice Guidelines for high-risk pediatric spine surgery that are applicable to AIS surgery. ${ }^{15}$ When hair is removed from the surgical site, clippers should be used as opposed to shaving. Impervious dressings are preferred and changes to the dressing should be minimized before discharge. Furthermore, they recommend routine intraoperative wound irrigation; both $>2,000 \mathrm{~mL}$ of normal saline and dilute betadine irrigation have been shown to reduce SSI after adult spine surgery, with neither solution proven to be superior. ${ }^{15}$

Several other factors may alter the risk of SSI in AIS surgery. In a systematic review, Vitale et al found that there is conflicting or poor-quality evidence that volume of blood loss, rates of blood transfusion, greater number of levels fused, and prolonged operative time increase the risk of SSI. ${ }^{15}$ There is also weak evidence that drain use reduces $\mathrm{SSI}^{24}$; however, there is also evidence that drain use does not impact complication rate or SSI. ${ }^{25}$ The authors' recommended infection prevention measures are summarized in Figure 1.

\section{Preventing neurologic injury}

Prevention of neurologic injury begins with safe patient positioning. Commonly, surgical correction of AIS requires

\begin{tabular}{|c|c|}
\hline Preadmission & Intraoperative \\
\hline $\begin{array}{l}\text { - Preoperative patient } \\
\text { education } \\
\text { - Preoperative } \\
\text { chlorohexidine } \\
\text { - Counseling and screening } \\
\text { for nutritional deficiency } \\
\text { and obesity }\end{array}$ & $\begin{array}{l}\text { - Limit operating room traffic } \\
\text { - Vancomycin powder in all } \\
\text { wounds } \\
\text { - Wound irrigation with } \\
>2,000 \mathrm{~mL} \text { of normal saline } \\
\text { - Reduce unnecessary } \\
\text { prolongation of surgical time } \\
\text { - Minimize allogenic blood } \\
\text { product administration } \\
\text { - Impervious dressing }\end{array}$ \\
\hline \multicolumn{2}{|c|}{$\begin{array}{l}\text { Preoperative } \\
\text { - Cefazolin } 20 \mathrm{mg} / \mathrm{kg} \text { (maximum 2,000 mg) given } 30 \text { minutes } \\
\text { prior to incision } \\
\text { - If minor penicillin/cephalosporin allergy, test dose cefazolin } \\
\text { - If serious penicillin/cephalosporin allergy, vancomycin } \\
10 \mathrm{mg} / \mathrm{kg} \text { given } 150 \text { minutes prior to incision } \\
\text { - Ensure appropriate timing, dosing, and redosing of antibiotics } \\
\text { - Hair removal with clippers }\end{array}$} \\
\hline
\end{tabular}

Figure I Perioperative interventions to reduce surgical site infections after surgical treatment of adolescent idiopathic scoliosis. posterior spinal fusion (PSF) and, thus, prone positioning. Complications from prone positioning for spinal surgery include perioperative blindness and peripheral neuropathies including brachial plexus injury.

In a retrospective review of over 500,000 patients undergoing spinal fusion, the incidence of perioperative blindness is 1.9 events per 10,000 cases. Diabetes, end-organ damage, spinal deformity surgery, and paralysis were independent risk factors for perioperative blindness, with over $56 \%$ of events occurring in spinal deformity surgeries. ${ }^{26}$ Perioperative blindness can result from several etiologies including ischemic optic neuropathy, central retinal artery occlusion (CRAO), and cortical blindness. ${ }^{27}$ Ischemic optic neuropathy results from decreased ocular profusion and is the most common cause of perioperative blindness. It is associated with obesity, male sex, Wilson frame use, longer anesthesia time, greater estimated blood loss, and fluid resuscitation with increased ratios of crystalloids to colloids. ${ }^{28} \mathrm{CRAO}$ is the second most common form of perioperative blindness and results from either direct or indirect pressure on the eye that increases intraocular pressure to the point of ischemia. CRAO, also known as headrest syndrome, is both a preventable and an irreversible form of blindness. ${ }^{29}$ Cortical blindness is caused by decreased perfusion to the visual cortex of the occipital lobes in the brain. This irreversible form of blindness is typically bilateral and associated with hypotension, prolonged hypoxia, cardiac arrest, and thromboembolic events. ${ }^{29}$ Prevention of perioperative blindness should focus on reducing the risk of prolonged hypotension, repletion of surgical blood loss, and avoidance of direct compression of the eyes. ${ }^{27,29}$

Peripheral nerve injuries are a rare perioperative complication with an estimated frequency of $0.03 \%{ }^{30}$ The most common sites of perioperative peripheral neuropathy are ulnar neuropathy, brachial plexus injury, median neuropathy, and radial neuropathy, accounting for $28 \%, 20 \%, 4 \%$, and $3 \%$ of all anesthesia-related nerve injury malpractice claims. ${ }^{31}$ Ulnar nerve injuries may result from direct compression over the cubital tunnel and/or excessive elbow flexion $>90^{\circ} .{ }^{27}$ The cervical and thoracic nerve roots of the brachial plexus are susceptible to injury as they pass superficially in the axilla around several bony prominences, tethered proximally at the vertebrae and distally by the axillary fascia. Improper positioning, specifically with the arm abducted $>90^{\circ}$, places excessive tension or compression on the brachial plexus and is associated with postoperative brachial plexopathy. ${ }^{32}$ Two retrospective reviews of over 800 prone positioned pediatric scoliosis surgery patients examined the rate of impending intraoperative brachial 
plexopathy using somatosensory cortical evoked potentials (SSEP). Between $3.6 \%$ and $6.2 \%$ of patients developed SSEP findings highly concerning for impending brachial plexopathy, with a reduction in SSEP amplitude of $>30 \% .33,34$ The use of neuromonitoring of the ulnar nerve with SSEP is effective for the early detection and prevention of brachial plexopathy related to patient positioning. Based on the current evidence, it is recommended that when patients are positioned prone, their arms should be abducted $<90^{\circ}$, the elbow should not be fully extended if the shoulders are abducted, and ulnar nerve SSEP should be utilized to prevent impending brachial plexus injuries. ${ }^{27,31-34}$ Care should also be taken when padding and positioning the pelvic bolsters to prevent compression on the lateral femoral cutaneous nerve. Lateral femoral cutaneous nerve neuropathy has been reported in up to $24 \%$ of patients undergoing posterior spine surgery, with longer surgical time being identified as a risk factor. ${ }^{35}$ The pain, hypersensitivity, paresthesia, or numbness typically resolves within 2-6 months, but may require steroid injections or decompression. ${ }^{27}$ In addition to preventing position-related neurologic injuries, all bony prominences should be padded to reduce the risk of pressure ulcerations. The abdomen should be allowed to hang freely; this has been shown to decrease intra-abdominal pressure, reduce the risk of abdominal compartment syndrome, and minimize blood loss during spinal surgery. ${ }^{27,31,36}$

Neurologic complication remains a devastating, yet rare complication after surgical correction of AIS. In 2005, the Scoliosis Research Society reported a $0.3 \%$ incidence of neurologic deficits after PSF and a $1.2 \%$ incidence after combined anterior and posterior surgery. ${ }^{4}$ Both the reduction in quality of life and financial costs related to neurogenic complications and spinal cord injury are enormous. The estimated lifetime cost of an injury resulting in paraplegia at age 25 is $\$ 977,142 .{ }^{37}$

Correction of spinal deformity places the spinal cord and the nerve roots at risk for injury, which may result in loss of motor and/or sensory function. Intraoperative monitoring of the neurologic status allows for early identification of possible neurologic injury and possible intervention to prevent such an injury. ${ }^{38}$ Successful implementation of neurophysiologic monitoring requires coordination of care between the surgeon, anesthesiologist, neurophysiologist, and operative staff. Despite the resources required for intraoperative monitoring, it still remains cost-effective. ${ }^{37}$ SSEP assess the functional status of the sensory tracts through stimulation of a peripheral nerve and record the electrical responses at various locations along that neural pathway. Other modalities such as neurogenic motor evoked potentials (NMEP) and transcranial motor evoked potentials (tcMEP) have been developed to monitor intraoperative dorsolateral and ventral motor tract function. ${ }^{39}$ Padburg et al demonstrated that the combined intraoperative use of SSEP and NMEP predicted neurologic status with $98.6 \%$ sensitivity, $100 \%$ specificity, a false-positive rate of $0.014 \%$, and no false negatives. They concluded that normal combined motor and sensory monitoring findings obviated the need for intraoperative wake-up tests, the gold standard method for intraoperative assessment of neurologic injury. ${ }^{40}$

Recent evidence has demonstrated that NMEP actually reflect a backfiring through the afferent sensory or dorsal column pathways, instead of testing the motor spinal pathways. ${ }^{41}$ In tcMEP monitoring, the response of electrical stimulation of the motor cortex through transcutaneous leads is recorded at the target muscle groups, which tests the native motor pathway more directly. ${ }^{42}$ Inhalation anesthetics can easily disrupt tcMEP; IV anesthesia may be a more favorable form of anesthesia for tcMEP monitoring. ${ }^{39}$ Level I evidence based on 1,121 patients undergoing surgery for AIS demonstrated the efficacy of combined SSEP and tcMEP monitoring in both detecting impending spinal cord injuries and allowing for interventions that may preserve spinal cord function. ${ }^{42}$ In 2014, Vitale et al released a list of "Best Practices" for intraoperative neuromonitoring. ${ }^{43}$ The consensus based on current evidence recommends the routine use of SSEP and tcMEP or other forms of motor tract monitoring, defines a $50 \%$ degradation of SEEP amplitude or a sustained decrease in tcMEP as a significant warning criterion, and suggests that wake-up tests should be considered when patients have persistent signal changes or cannot be monitored ${ }^{43}$ Furthermore, Vitale et al recommended the implementation of a multidisciplinary checklist for how to respond to concerning neuromonitoring changes, which focuses on communication between the surgeon, anesthesiologist, perioperative nursing staff, and the neurophysiology team.

The development of intraoperative evoked electromyography (EMG) monitoring of pedicle screws provides a safe and effective way to evaluate for iatrogenic neurologic injury from aberrant screw placement. Glassman et al demonstrated that a normal EMG response predicts the absence of nerve injury, while an abnormal EMG response may or may not predict neurologic deficit requiring further clinical or radiographic investigation. ${ }^{44}$ Figure 2 summarizes the authors' recommended interventions to reduce the risk of perioperative neurologic injury. 


\section{Preventing neurologic injury}

- Appropriate patient positioning with arms at $<90^{\circ}$ of abduction and $90^{\circ}$ of flexion

- Padding and protection of the eyes, head, and other pressure points during prone positioning

- Intraoperative SSEP and tcMEP

- Review preoperatively the plan of action with the surgical team if neuromonitoring changes of concern occur

- Evoked EMG monitoring of pedicle screws during implantation

- MAP of $>65 \mathrm{mmHg}$ during surgery with higher pressure goals (ie, $76 \mathrm{mmHg}$ ) during correction maneuvers

Figure 2 Recommended interventions to prevent neurologic injury during posterior spinal fusion for adolescent idiopathic scoliosis.

Abbreviations: EMG, electromyography; MAP, mean arterial pressure; SSEP, somatosensory cortical evoked potentials; tcMEP, transcranial motor evoked potentials.

\section{Reducing perioperative blood loss}

Soft tissue dissection, bone decortication, osteotomies, and instrumentation involved with surgical correction of AIS can lead to significant blood loss. Blood loss during AIS surgery varies significantly, with the average blood loss during PSF ranging from 275 to $907 \mathrm{~mL} .{ }^{45-47}$ Also, estimates for blood loss during anterior spinal fusion average $323 \pm 171 \mathrm{~mL}$ and during combined anterior and posterior approaches average $1,277 \pm 821 \mathrm{~mL} .{ }^{46}$ Operative time, male sex, and increased preoperative kyphosis have been identified as independent predictors of increased blood loss. ${ }^{46}$ Excessive perioperative blood loss places patients at risk for infection, hemodynamic instability, cardiopulmonary dysfunction, renal failure, and possible death. Also, allogenic blood transfusion presents additional risks for patients, including potential blood-borne infection, transfusion reaction, electrolyte imbalance, coagulopathy, and increased risk of SSI. ${ }^{46,48-50}$ Allogenic blood transfusion has been associated with complications in up to $20 \%$ of patients undergoing spinal surgery. ${ }^{51}$ Certain factors place patients at risk for needing allogenic red blood cell transfusion, including higher American Society of Anesthesiologists physical status classification, longer surgical duration, and increased number of levels fused..$^{52}$ Minimizing perioperative blood loss and reducing the need for allogenic blood products are essential for safety in AIS surgery.

Controlled hypotensive anesthesia utilizes pharmacologic agents in order to maintain a mean arterial pressure (MAP) below normal physiologic levels. Deliberate hypotension has been shown to be a safe and effective method to reduce operative time and surgical blood loss in spine surgery as well as total joint arthroplasty. ${ }^{46,49,53,54}$ A retrospective analysis of over 300 AIS patients found that by lowering MAPs to $<65 \mathrm{mmHg}$ at the time of incision reduced blood loss by $33 \%$ with no complications related to the use of hypotension. ${ }^{49}$ When blood pressure levels were elevated at the time of incision, blood loss increased by $29 \%$ and operative time was 29 minutes greater than the hypotensive anesthesia cohort. Hypotensive anesthesia with an ideal MAP $<65 \mathrm{mmHg}$ at the time of incision minimizes surgical blood loss, improves visualization during exposure of the spine, and reduces operative times. Short-acting neuromuscular blockade with agents such as rocuronium may also decrease blood loss during exposure, but should be given early enough in the dissection so as to allow neuromonitoring to resume prior to instrumentation.

Lowering MAP during the surgical approach in order to reduce intraoperative blood loss must be balanced with maintaining a MAP necessary for spinal cord perfusion. MAP $<60 \mathrm{mmHg}$ during correction of spinal deformity has been associated with significant SSEP changes and an increased risk of spinal cord ischemia. ${ }^{55}$ While high-quality data on specific MAP goals during the instrumentation and correction of deformity is lacking, it is recommended that the MAP should be $>70 \mathrm{mmHg}$ during this portion of the case ${ }^{56,57}$ The MAP should be elevated further to a goal of $>80$ $\mathrm{mmHg}$ if there is high concern for neurologic injury, such as a complete loss of neuromonitoring signals. ${ }^{57}$

Antifibrinolytic agents such as tranexamic acid and $\varepsilon$-aminocaproic acid are widely used perioperatively in surgical procedures with the potential for significant blood loss. These medications are synthetic analogs of lysine, which reversibly block lysine-binding sites on plasminogen, preventing the activation of plasmin and inhibiting fibrinolysis. ${ }^{58}$ Several prospective, randomized, double-blind, placebo-controlled studies show that tranexamic acid is an effective, safe, and cheap method to reduce blood loss during spinal fusion. ${ }^{59-61}$ Verma et al found that both tranexamic acid and $\varepsilon$-aminocaproic acid reduced operative blood loss, but not transfusion rate. ${ }^{60}$ Tranexamic acid was more effective in reducing blood loss. Of note, there was no difference between treatment and placebo groups when the MAP was $>75 \mathrm{mmHg}$, suggesting the importance of MAP in surgical blood loss. ${ }^{60}$ Dosing of tranexamic acid varies with loading doses ranging from 10 to $30 \mathrm{mg} / \mathrm{kg}$ and subsequent continuous infusion dosing from 1 to $10 \mathrm{mg} / \mathrm{kg} / \mathrm{h} \cdot{ }^{48,59,60}$ A 2008 Cochrane Review of the effectiveness of antifibrinolytic drugs on blood loss in children undergoing scoliosis surgery concluded that these agents are indeed effective in reducing surgical blood loss, but the effect on decreasing blood transfusion remains unclear. ${ }^{62}$

Cell salvage systems (cell saver) can be used intraoperatively to collect blood from the surgical field, filter cellular, noncellular, and biochemical debris, and provide a source of autologous red blood cell transfusion. ${ }^{63}$ The use of cell saver 


Reducing surgical blood loss
- Hypotensive anesthesia with MAP goals of $65 \mathrm{mmHg}$ at the
time of incision
- Administration of antifibrinolytic agents (tranexamic acid
loading dose $10 \mathrm{mg} / \mathrm{kg}$ prior to incision and continue infusion
dose of $1 \mathrm{mg} / \mathrm{kg} / \mathrm{h}$ until closure)
- Routine use of cell salvage system
- Delay decortication until the end of the case

Figure 3 Recommended intraoperative measures to reduce surgical blood loss. Abbreviations: MAP, mean arterial pressure.

has been shown to reduce the need for allogenic transfusion in pediatric pelvic and spine surgery. ${ }^{63-65}$ While autologous transfusion is exceptionally safe, there are reports of rare transfusion reactions. ${ }^{66} \mathrm{~A}$ retrospective case-control study found that cell saver use with a $150 \mathrm{~mL}$ collection bowl reduced allogenic transfusion rates and volumes during PSF for AIS. ${ }^{63}$ Cell saver was more effective in surgeries lasting longer than 6 hours and with estimated blood losses of $>30 \%$ of total blood volume. Other studies have questioned the need for routine use of cell saver in AIS surgery. ${ }^{67,68} \mathrm{In}$ a nonrandomized prospective study of 95 children undergoing PSF for AIS, Weiss et al found that the use of cell saver did not reduce the risk of allogenic transfusion. ${ }^{67}$ Their cell saver system required $250 \mathrm{~mL}$ of blood collected in order to be washed and returned, as opposed to the $150 \mathrm{~mL}$ required in other studies. ${ }^{63,67}$ Figure 3 outlines the measures recommended to reduce perioperative blood loss.

\section{Reducing postoperative complications}

In order to expedite discharge and return patients to a feeling of "normalcy" after surgery, it is essential to prevent and manage any medical complications. Certain AIS patients are at greater risk of sustaining a complication after surgery. A retrospective review of over 700 AIS patients who underwent PSF in 2012 found that those children with a body mass index in the 95th percentile or greater were significantly more likely to have an adverse event after PSF. Patients who had $>13$ levels instrumented or operative times exceeding 365 minutes were more likely to have an extended length of stay in hospital ( $>6$ days). ${ }^{69}$ Pugely et al investigated the factors that contributed to short-term mortality after spinal fusion and found that those patients with cognitive impairment, elevated American Society of Anesthesiologists classification, a history of hepatobiliary disease, prolonged operative times, and fusion to the pelvis had significantly higher complication rates. ${ }^{70}$ Particular attention should be paid to patients with the above risk factors in order to minimize the complication rates.
The idea of utilizing postoperative protocols to reduce complications is not new. Wenger et al published a review article in 1992 which proposed a goal of a routine 5-7 day postoperative stay for straightforward AIS patients undergoing PSF. ${ }^{71}$ This review provided recommendations that are also included in modern accelerated discharge (AD) pathways, such as frequent incentive spirometer use, elevating the head of patients' beds to allow for better lung expansion, early Foley removal to minimize the risk of urinary tract infections, and having a low threshold for aspirating fluid collections or hematomas, to aid in early diagnosis and treatment of wound issues. Minimizing perioperative blood loss may help prevent postoperative orthostasis which can contribute to delayed mobilization and slow recovery. Monitoring patients' wounds postoperatively can provide early signs of a developing wound hematoma or SSI. Temperature spikes are very common in the immediate postoperative period; $72 \%$ of patients had fever with temperatures $>38^{\circ} \mathrm{C}$ and $9 \%$ had temperatures $>39^{\circ}$ in one study. ${ }^{72}$ However, this has not been correlated to postoperative infection. Fevers after postoperative day (POD) 3 or 4 are likely of greater concern and should be evaluated with appropriate cultures. Another major source of infection is the urinary tract, and any organisms found in a patient's urine before or after surgery should be treated aggressively. ${ }^{15}$

Ileus is a common complication after spinal fusion surgery, with an incidence of between $0.6 \%$ and $16.7 \%$ in adult lumbar spine surgery. ${ }^{73}$ Nachlas et al studied gastric, intestinal, and colonic motility by tracking the barium administered immediately postop in 160 patients in a landmark study performed in 1972. They found that, in patients having extraintestinal procedures, gastric motility returned within 24 hours, and colonic motility can be slowed for 3-5 days but return can be hastened by giving a laxative. ${ }^{74} \mathrm{An}$ aggressive bowel regimen is important for postoperative bowel function. In the $\mathrm{AD}$ pathway, following surgery, all patients are allowed to eat ice chips and transitioned to a clear liquid diet on POD0 followed by initiation of solid foods on POD1. While patients' appetite may take several days to return in full, most children easily tolerate small meals starting on POD1 with minimal nausea. Unlike neuromuscular scoliosis patients, most AIS patients are well-nourished at baseline. Thus, while early resumption of an oral diet provides the much needed nutrition to patients during the early healing period, it more importantly allows for dosing of oral pain medications on POD1. Oral narcotics and antispasmodics tend to be longer acting with less sedating and other adverse side effects. Discontinuation of the patient-controlled analgesia also eliminates an additional impediment to mobilization. 
Pulmonary complications can contribute to prolonged hospital stays, especially among patients with pre-existing pulmonary issues. Scoliosis is associated with progressive restrictive lung disease, and thoracic surgery impedes respiration due to postoperative pain, anesthesia, and immobilization. Pneumonia, respiratory failure requiring prolonged mechanical ventilation, bronchospasm, and atelectasis are the common complications seen following PSF; however, these pulmonary complications are much more common among neuromuscular patients. ${ }^{75}$ Nonetheless, AIS patients with a history of poor exercise tolerance, a curve exceeding $80^{\circ}$, or a history of severe reactive airway disease stand to benefit from a preoperative pulmonary evaluation. ${ }^{76} \mathrm{~A}$ retrospective review of pediatric patients who had pulmonary function testing prior to spinal fusion demonstrated that patients with preoperative forced expiratory volume in 1 second $<40 \%$ predicted, vital capacity $<60 \%$, inspiratory capacity $<30 \mathrm{~mL} / \mathrm{kg}$, or total lung capacity $<60 \%$ were more likely to require prolonged postoperative mechanical ventilation than those patients who did not have them. ${ }^{76}$ Preoperative testing can provide vital insight and allow for better planning on behalf of both physicians and patients' families.

While the majority of AIS patients are otherwise healthy in contrast to the neuromuscular scoliosis patient population, a minority of AIS patients have severe medical comorbidities. This subpopulation of medically complex AIS patients merits more extensive preoperative workup and optimization. This may include hyperalimentation and nutritional supplementation, pulmonary function testing, cardiac assessment, and the assistance of a pediatric hospitalist both prior to and after surgery to streamline care. ${ }^{77}$ While these patients stand to benefit from many of the principles included in the pathway, especially early mobilization, this population of patients is not the target of the AD pathway.

\section{Optimizing postoperative care}

Treating children with AIS is a complex undertaking. Traditionally, hospital stays after spinal fusion procedures have been prolonged. Factors that have historically contributed to length of stay are difficulty with mobilization, pain control, resumption of general diet, and residual drain output. A multidisciplinary approach that addresses these factors in hopes that an earlier return to "normalcy" both expedites discharge and reduces the complications of prolonged immobilization and lengthy hospital stays. ${ }^{45}$

The author's recommended AD protocol truly begins prior to surgery, as expectations must be explained to patients and their families (Figure 4). Patients' caregivers are informed that their children will be rapidly mobilized to allow for a short hospital stay. The importance of preoperative counseling cannot be underestimated, as many families expect prolonged hospital stays after surgery. Knowing that the patients will likely be discharged as soon as 1-2 days after surgery allows the caregivers to plan appropriately for their return home. A team-based approach to family reassurance during the hospitalization is also critical and all team members need to understand the expectations for AD.

The importance of early mobilization after spine surgery has been emphasized by a number of studies. This idea was proposed as early as 1973, when Leider et al proposed that AIS patients should be encouraged to ambulate early after surgery, citing that "rapid mobilization reduces fatigue and allows more rapid return to a more normal life-style". ${ }^{78}$ The next several decades witnessed marked improvements in instrumentation systems for spinal fusion. In 1988, Heilbronner and Sussman performed an early investigation of 40 AIS patients who had undergone Harrington rod instrumentation. The goal of new spinal instrumentation systems was, in fact, to rid the need for body casts and prolonged immobilization. At that time, the length of stay ranged from 5 to 13 days and averaged 8.5 days. ${ }^{79}$ This trend has continued, as instrumentation has continued to improve and provide more robust fixation to allow for faster and more extensive mobilization. A survey of Shriners hospital spinal deformity surgeons published in 2007 revealed that, within the Shriners system, therapy for AIS patients was aimed at moving patients early, with the goal of sitting on POD1, standing on POD2, and walking on POD2 or $3 .{ }^{80}$ Given the fact that the majority of AIS patients are otherwise healthy, high-functioning children preoperatively, returning them to their preoperative level of function or better in a timely fashion is critically important. Tarrant et al's prospective study of AIS patients undergoing PSF found that, on average, these patients return to school or college full-time 10 weeks postoperatively, and over $50 \%$ returned to unrestrained physical activity by 24 weeks postop. ${ }^{81}$ This is markedly faster than in past decades, and many children may be able to surpass these goals. The AD protocol aims to motivate and enable children to return to their daily activities as soon as possible.

Pain control regimens vary between institutions. The AD protocol calls for the use of a patient-controlled analgesia and IV benzodiazepines on the night of POD0 with an option to include gabapentin and ketorolac. These IV medications are discontinued on the morning of POD1, assuming patients are tolerating an oral diet, with only breakthrough doses of IV narcotic continued as needed beyond this point. An 


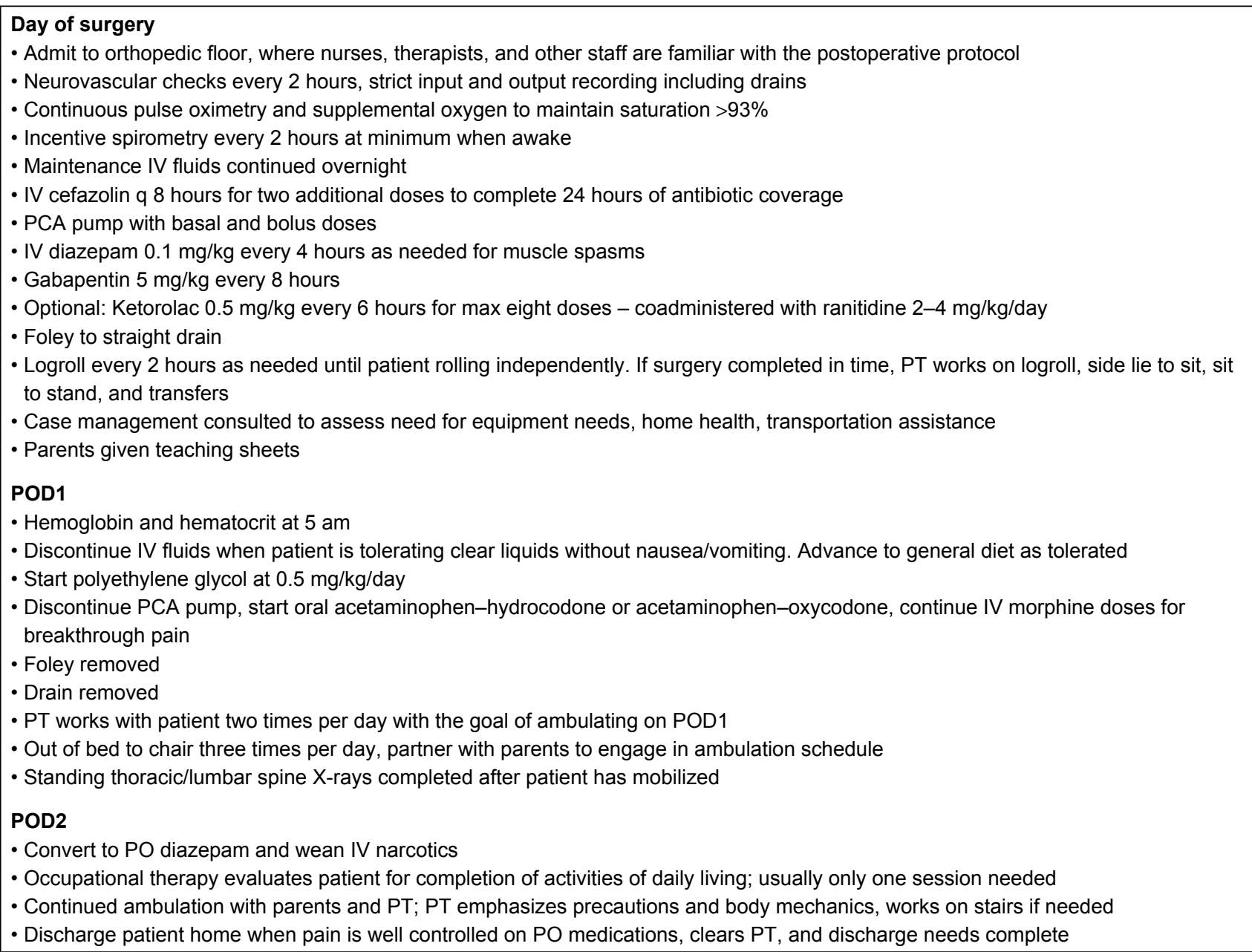

Figure 4 Postoperative protocol for patients undergoing posterior spinal fusion for adolescent idiopathic scoliosis.

Abbreviations: IV, intravenous; PCA, patient-controlled analgesia; POD, postoperative day; PT, physical therapy.

acute pain service consult is placed only for those patients with an extensive chronic pain management history or those who have difficulty with pain despite the above regimen, and this is a rare occurrence. Epidural catheters and intrathecal injections have been evaluated extensively for their use in pain control, but these are not commonly employed as part of the AD protocol. ${ }^{20,82-84} \mathrm{IV}$ acetaminophen is often used as an adjunct in multimodal pain regimen. While it is still not a routinely given medication due to availability and cost, it can be given while the patient is fasting; it is nonsedating and may serve to decrease narcotic requirements. ${ }^{85}$

Wound drains and Foley catheters are all removed on POD1 in the AD protocol. The potential advantages and disadvantages of using drains after PSF have been evaluated in several studies. Theoretically, drains promote egress of fluids away from the cutaneous incision and minimize the formation of hematoma which can serve as a medium for bacterial growth; however, they also create the potential for drain contamination, impeded mobilization, increased discomfort, and increased care needs (ie, drain stripping or reservoir emptying). Diab et al evaluated 324 drained patients and 176 undrained patients and found that the mean time to drain removal was 57 hours, with half of the surgeons using drain output as a criterion to determine when to remove their drains and the other half leaving them for a predetermined amount of time (15 surgeons removed the drain after 48 hours, 2 after 72 hours, and 1 after 24 hours). ${ }^{25}$ Length of stay in hospital did not differ between these two groups. They found that drain use was beneficial, but no consensus was found regarding how long to leave drains in place. Clearly, only a minority of surgeons removed their drains during the first 24 hours after surgery. Blank et al randomized drain use in 30 AIS patients undergoing PSF. ${ }^{86}$ Dressings were examined at 4, 12, 24, and 48 hours postoperatively, and all drains were removed at 48 hours. They found that closed suction reduced the frequency of needed dressing changes, and it also reduced the rate of wound complications including superficial hematoma or infection 
and the ultimate need for implant removal. ${ }^{86}$ Early drain removal on POD1 has been found to expedite mobilization and decrease patient discomfort without a resultant increase in wound complications. ${ }^{45}$

\section{Summary}

The surgical care for AIS patients requires a multidisciplinary approach to minimize complications and maximize outcomes. Multidisciplinary teams have successfully been shown to improve the quality of care for AIS patients by reducing infection rates. ${ }^{5}$ There are several evidence-based methods which have been shown to reduce the risk of infection, prevent neurologic injury, minimize surgical blood loss, and optimize postoperative care. It is the responsibility of every member of the perioperative team to take ownership of the patient and to be actively involved in improving patient care.

\section{Acknowledgments}

Medtronic Spine, Zimmer/Biomet, and Orthopaediatric are acknowledged for the consulting services. Harrison Foundation is acknowledged for the grant support.

\section{Disclosure}

The authors report no conflicts of interest in this work.

\section{References}

1. Weinstein SL, Dolan LA, Cheng JCY, Danielsson A, Morcuende JA Adolescent idiopathic scoliosis. Lancet. 2008;371(9623):1527-1537.

2. Asher MA, Burton DC. Adolescent idiopathic scoliosis: natural history and long term treatment effects. Scoliosis. 2006;1(1):2.

3. Dolan LA, Weinstein SL. Surgical rates after observation and bracing for adolescent idiopathic scoliosis: an evidence-based review. Spine (Phila Pa 1976). 2007;32(19 Suppl):S91-S100.

4. Coe JD, Arlet V, Donaldson W, et al. Complications in spinal fusion for adolescent idiopathic scoliosis in the new millennium. A report of the Scoliosis Research Society Morbidity and Mortality Committee. Spine (Phila Pa 1976). 2006;31(3):345-349.

5. Ballard MR, Miller NH, Nyquist AC, Elise B, Baulesh DM, Erickson MA. A multidisciplinary approach improves infection rates in pediatric spine surgery. J Pediatr Orthop. 2012;32(3):266-270.

6. Li Y, Glotzbecker M, Hedequist D. Surgical site infection after pediatric spinal deformity surgery. Curr Rev Musculoskelet Med. Epub 2012 Feb 9.

7. Whitehouse JD, Friedman ND, Kirkland KB, Richardson WJ, Sexton DJ. The impact of surgical-site infections following orthopedic surgery at a community hospital and a university hospital: adverse quality of life, excess length of stay, and extra cost. Infect Control Hosp Epidemiol. 2002;23(4):183-189.

8. Hedequist D, Haugen A, Hresko T, Emans J. Failure of attempted implant retention in spinal deformity delayed surgical site infections. Spine (Phila Pa 1976). 2009;34(1):60-64.

9. Popovich KJ, Lyles R, Hayes R, Hota B, Trick W, Weinstein RA, Hayden MK. Relationship between chlorhexidine gluconate skin concentration and microbial density on the skin of critically ill patients bathed daily with chlorhexidine gluconate. Infect Control Hosp Epidemiol. 2012; 33(9):889-896.
10. Johnson AJ, Kapadia BH, Daley JA, Molina CB, Mont MA. Chlorhexidine reduces infections in knee arthroplasty. J Knee Surg. 2013;26(3): 213-218.

11. Kapadia BH, Johnson AJ, Daley JA, Issa K, Mont MA. Pre-admission cutaneous chlorhexidine preparation reduces surgical site infections in total hip arthroplasty. J Arthroplasty. 2013;28(3):490-493.

12. Jevsevar DS, Karlin LI. The relationship between preoperative nutritional status and complications after an operation for scoliosis in patients who have cerebral palsy. J Bone Joint Surg Am. 1993;75(6): 880-884.

13. Croft LD, Pottinger JM, Chiang HY, Ziebold CS, Weinstein SL, Herwaldt LA. Risk factors for surgical site infections after pediatric spine operations. Spine (Phila Pa 1976). 2015;40(2):E112-E119.

14. Linam WM, Margolis PA, Staat MA, Britto MT, Hornung R, Cassedy A, Connelly BL. Risk factors associated with surgical site infection after pediatric posterior spinal fusion procedure. Infect Control Hosp Epidemiol. 2009;30(2):109-116.

15. Vitale MG, Riedel MD, Glotzbecker MP, et al. Building consensus: development of a Best Practice Guideline (BPG) for surgical site infection (SSI) prevention in high-risk pediatric spine surgery. J Pediatr Orthop. 2013;33(5):471-478.

16. Transfeldt E, Lonstein J, Winter R, Bradford D, Moe J, Mayfield J. Wound infections in reconstructive spinal surgery. Orthop Trans. 1985; 9:128.

17. Myung KS, Glassman DM, Tolo VT, Skaggs DL. Simple steps to minimize spine infections in adolescent idiopathic scoliosis. $J$ Pediatr Orthop. 2014;34(1):29-33.

18. Labbe AC, Demers AM, Rodrigues R, Arlet V, Tanguay K, Moore DL. Surgical-site infection following spinal fusion: a case-control study in a children's hospital. Infect Control Hosp Epidemiol. 2003;24(8): 591-595.

19. Rosenberg AD, Wambold D, Kraemer L, et al. Ensuring appropriate timing of antimicrobial prophylaxis. J Bone Joint Surg Am. 2008; 90(2):226-232.

20. Borkhuu B, Borowski A, Shah SA, Littleton AG, Dabney KW, Miller F. Antibiotic-loaded allograft decreases the rate of acute deep wound infection after spinal fusion in cerebral palsy. Spine (Phila Pa 1976). 2008; 33(21):2300-2304.

21. Sweet FA, Roh M, Sliva C. Intrawound application of vancomycin for prophylaxis in instrumented thoracolumbar fusions: efficacy, drug levels, and patient outcomes. Spine (Phila Pa 1976). 2011;36(24):2084-2088.

22. O'Neill KR, Smith JG, Abtahi AM, Archer KR, Spengler DM, McGirt MJ, Devin CJ. Reduced surgical site infections in patients undergoing posterior spinal stabilization of traumatic injuries using vancomycin powder. Spine J. 2011;11(7):641-646.

23. Panahi P, Stroh M, Casper DS, Parvizi J, Austin MS. Operating room traffic is a major concern during total joint arthroplasty. Clin Orthop Relat Res. 2012;470(10):2690-2694.

24. Ho C, Sucato DJ, Richards BS. Risk factors for the development of delayed infections following posterior spinal fusion and instrumentation in adolescent idiopathic scoliosis patients. Spine (Phila Pa 1976). 2007; 32(20):2272-2277.

25. Diab M, Smucny M, Dormans JP, et al. Use and outcomes of wound drain in spinal fusion for adolescent idiopathic scoliosis. Spine (Phila Pa 1976). 2012;37(11):966-973.

26. Nandyala SV, Marquez-Lara A, Fineberg SJ, Singh R, Singh K. Incidence and risk factors for perioperative visual loss after spinal fusion. Spine J. 2014;14(9):1866-1872.

27. DePasse JM, Palumbo MA, Haque M, Eberson CP, Daniels AH. Complications associated with prone positioning in elective spinal surgery. World J Orthop. 2015;6(3):351-359.

28. Postoperative Visual Loss Study G. Risk factors associated with ischemic optic neuropathy after spinal fusion surgery. Anesthesiology. 2012;116(1):15-24.

29. Stambough JL, Dolan D, Werner R, Godfrey E. Ophthalmologic complications associated with prone positioning in spine surgery. $J$ Am Acad Orthop Surg. 2007;15(3):156-165. 
30. Welch MB, Brummett CM, Welch TD, Tremper KK, Shanks AM, Guglani P, Mashour GA. Perioperative peripheral nerve injuries: a retrospective study of 380,680 cases during a 10-year period at a single institution. Anesthesiology. 2009;111(3):490-497.

31. Kamel I, Barnette R. Positioning patients for spine surgery: Avoiding uncommon position-related complications. World J Orthop. 2014; 5(4):425-443.

32. Uribe JS, Kolla J, Omar H, Dakwar E, Abel N, Mangar D, Camporesi E. Brachial plexus injury following spinal surgery. J Neurosurg Spine. 2010;13(4):552-558.

33. Schwartz DM, Drummond DS, Hahn M, Ecker ML, Dormans JP. Prevention of positional brachial plexopathy during surgical correction of scoliosis. J Spinal Disord. 2000;13(2):178-182.

34. Labrom RD, Hoskins M, Reilly CW, Tredwell SJ, Wong PK. Clinical usefulness of somatosensory evoked potentials for detection of brachial plexopathy secondary to malpositioning in scoliosis surgery. Spine (Phila Pa 1976). 2005;30(18):2089-2093.

35. Yang $\mathrm{SH}, \mathrm{Wu} \mathrm{CC}$, Chen $\mathrm{PQ}$. Postoperative meralgia paresthetica after posterior spine surgery: incidence, risk factors, and clinical outcomes. Spine (Phila Pa 1976). 2005;30(18):E547-E550.

36. Kwee MM, Ho YH, Rozen WM. The prone position during surgery and its complications: a systematic review and evidence-based guidelines. Int Surg. 2015;100(2):292-303.

37. Sala F, Dvorak J, Faccioli F. Cost effectiveness of multimodal intraoperative monitoring during spine surgery. Eur Spine J. 2007;16 Suppl 2:S229-S231.

38. Nash CL, Jr., Lorig RA, Schatzinger LA, Brown RH. Spinal cord monitoring during operative treatment of the spine. Clin Orthop Relat Res. 1977(126): 100-105.

39. Neuromonitoring information statement. SRS information statement, 2009; 2009. Available from: https://www.srs.org/about-srs/quality-andsafety/position-statements/neuromonitoring-information-statement. Accessed February 24, 2016.

40. Padburg AM, Wilson-Holden TJ, Lenke LG., Bridwell KH. Somatosensory-and motor-evoked potential monitoring without a wake-up test during idiopathic scoliosis surgery: an accepted standard of care. Spine (Phila Pa 1976). 1998;23(12):1392-1400.

41. Su CF, Haghighi SS, Oro JJ, Gaines RW. "Backfiring" in spinal cord monitoring. High thoracic spinal cord stimulation evokes sciatic response by antidromic sensory pathway conduction, not motor tract conduction. Spine (Phila Pa 1976). 1992;17(5):504-508.

42. Schwartz DM, Auerbach JD, Dormans JP, et al. Neurophysiological detection of impending spinal cord injury during scoliosis surgery. $J$ Bone Joint Surg Am. 2007;89(11):2440-2449.

43. Vitale MG, Skaggs DL, Pace GI, et al. Best practices in intraoperative neuromonitoring in spine deformity surgery: development of an intraoperative checklist to optimize response. Spine Deformity. 2014; 2(5):333-339.

44. Glassman SD, Dimar JR, Puno RM, Johnson JR, Shields CB, Linden $\mathrm{RD}$. A prospective analysis of intraoperative electromyographic monitoring of pedicle screw placement with computed tomographic scan confirmation. Spine (Phila Pa 1976). 1995;20(12):1375-1379.

45. Fletcher ND, Shourbaji N, Mitchell PM, Oswald TS, Devito DP, Bruce RW. Clinical and economic implications of early discharge following posterior spinal fusion for adolescent idiopathic scoliosis. $J$ Child Orthop. 2014;8(3):257-263.

46. Ialenti MN, Lonner BS, Verma K, Dean L, Valdevit A, Errico T. Predicting operative blood loss during spinal fusion for adolescent idiopathic scoliosis. J Pediatr Orthop. 2013;33(4):372-376.

47. Fletcher ND, Andras LM, Lazarus DE, et al. Use of a novel pathway for early discharge was associated with a $48 \%$ shorter length of stay after posterior spinal fusion for adolescent idiopathic scoliosis. J Pediatr Orthop. Epub 2015 July 24.

48. Grant JA, Howard J, Luntley J, Harder J, Aleissa S, Parsons D. Perioperative blood transfusion requirements in pediatric scoliosis surgery: the efficacy of tranexamic acid. J Pediatr Orthop. 2009;29(3): 300-304.
49. Verma K, Lonner B, Dean L, Vecchione D, Lafage V. Reduction of mean arterial pressure at incision reduces operative blood loss in adolescent idiopathic scoliosis. Spine Deformity. 2013;1(2):115-122.

50. Schwarzkopf R, Chung C, Park JJ, Walsh M, Spivak JM, Steiger D. Effects of perioperative blood product use on surgical site infection following thoracic and lumbar spinal surgery. Spine (Phila Pa 1976). 2010; 35(3):340-346.

51. Tate Jr DE, Friedman RJ. Blood conservation in spinal surgery: review of current techniques. Spine. 1992;17(12):1450-1456.

52. Wong J, El Beheiry H, Rampersaud YR, et al. Tranexamic acid reduces perioperative blood loss in adult patients having spinal fusion surgery. Anesth Analg. 2008;107(5):1479-1486.

53. Lawhon SM, Kahn A, 3rd, Crawford AH, Brinker MS. Controlled hypotensive anesthesia during spinal surgery. A retrospective study. Spine (Phila Pa 1976). 1984;9(5):450-453.

54. Paul JE, Ling E, Lalonde C, Thabane L. Deliberate hypotension in orthopedic surgery reduces blood loss and transfusion requirements: a meta-analysis of randomized controlled trials. Can J Anaesth. 2007; 54(10):799-810.

55. Owen JH. The application of intraoperative monitoring during surgery for spinal deformity. Spine. 1999;24(24):2649.

56. Mooney III JF, Bernstein R, Hennrikus Jr WL, MacEwen GD. Neurologic risk management in scoliosis surgery. J Pediatr Orthop. 2002; 22(5):683-689.

57. Pahys JM, Guille JT, D’Andrea LP, Samdani AF, Beck J, Betz RR. Neurologic injury in the surgical treatment of idiopathic scoliosis: guidelines for assessment and management. J Am Acad Orthop Surg. 2009; 17(7):426-434.

58. Ortmann E, Besser MW, Klein AA. Antifibrinolytic agents in current anaesthetic practice. Br J Anaesth. 2013;111(4):549-563.

59. Elwatidy S, Jamjoom Z, Elgamal E, Zakaria A, Turkistani A, El-Dawlatly A. Efficacy and safety of prophylactic large dose of tranexamic acid in spine surgery: a prospective, randomized, double-blind, placebocontrolled study. Spine (Phila Pa 1976). 2008;33(24):2577-2580.

60. Verma K, Errico T, Diefenbach C, et al. The relative efficacy of antifibrinolytics in adolescent idiopathic scoliosis: a prospective randomized trial. J Bone Joint Surg Am. 2014;96(10):e80.

61. Sethna NF, Zurakowski D, Brustowicz RM, Bacsik J, Sullivan LJ, Shapiro F. Tranexamic acid reduces intraoperative blood loss in pediatric patients undergoing scoliosis surgery. Anesthesiology. 2005;102(4): 727-732.

62. Tzortzopoulou A, Cepeda MS, Schumann R, Carr DB. Antifibrinolytic agents for reducing blood loss in scoliosis surgery in children. Cochrane Database Syst Rev. 2008(3):CD006883.

63. Bowen RE, Gardner S, Scaduto AA, Eagan M, Beckstead J. Efficacy of intraoperative cell salvage systems in pediatric idiopathic scoliosis patients undergoing posterior spinal fusion with segmental spinal instrumentation. Spine (Phila Pa 1976). 2010;35(2):246-251.

64. Lennon RL, Hosking MP, Gray JR, Klassen RA, Popovsky MA, Warner MA. The effects of intraoperative blood salvage and induced hypotension on transfusion requirements during spinal surgical procedures. Mayo Clin Proc. 1987;62(12):1090-1094.

65. Nicolai P, Leggetter PP, Glithero PR, Bhimarasetty CR. Autologous transfusion in acetabuloplasty in children. J Bone Joint Surg Br. 2004; 86(1):110-112.

66. Domen RE. Adverse reactions associated with autologous blood transfusion: evaluation and incidence at a large academic hospital. Transfusion. 1998;38(3):296-300.

67. Weiss JM, Skaggs D, Tanner J, Tolo V. Cell saver: is it beneficial in scoliosis surgery? J Child Orthop. 2007;1(4):221-227.

68. Copley LA, Richards BS, Safavi FZ, Newton PO. Hemodilution as a method to reduce transfusion requirements in adolescent spine fusion surgery. Spine (Phila Pa 1976). 1999;24(3):219-222.

69. Basques BA, Bohl DD, Golinvaux NS, Smith BG, Grauer JN. Patient factors are associated with poor short-term outcomes after posterior fusion for adolescent idiopathic scoliosis. Clin Orthop Relat Res. 2015; 473(1):286-294. 
70. Pugely AJ, Martin CT, Gao Y, Ilgenfritz R, Weinstein SL. The incidence and risk factors for short-term morbidity and mortality in pediatric deformity spinal surgery: an analysis of the NSQIP pediatric database. Spine (Phila Pa 1976). 2014;39(15):1225-1234.

71. Wenger DR, Mubarak SJ, Leach J. Managing complications of posterior spinal instrumentation and fusion. Clin Orthop Relat Res. 1992; 284:24-33.

72. Blumstein GW, Andras LM, Seehausen DA, Harris L, Ross PA, Skaggs DL. Fever is common postoperatively following posterior spinal fusion infection is an uncommon cause. J Pediatr. 2015;166(3):751-755.

73. Kiely PD, MountLE, DuJY, etal. Theincidence andrisk factors forpost-operative ileus after spinal fusion surgery: a multivariate analysis. Int Orthop 2016:40(6):1-8

74. Nachlas MM, Younis MT, Roda CP, Wityk JJ. Gastrointestinal motility studies as a guide to postoperative management. Ann Surg. 1972; 175(4):510.

75. Kang GR, Suh SW, Lee IO. Preoperative predictors of postoperative pulmonary complications in neuromuscular scoliosis. J Orthop Sci. 2011; 16(2):139-147.

76. Yuan N, Skaggs DL, Dorey F, Keens TG. Preoperative predictors of prolonged postoperative mechanical ventilation in children following scoliosis repair. Pediatr Pulmonol. 2005;40(5):414-419.

77. Rappaport DI, Adelizzi-Delany J, Rogers KJ, et al. Outcomes and costs associated with hospitalist comanagement of medically complex children undergoing spinal fusion surgery. Hosp Pediatr. 2013;3(3): 233-241.

78. Leider LL Jr., Moe JH, Winter RB. Early ambulation after the surgical treatment of idiopathic scoliosis. J Bone Joint Surg Am. 1973;55(5): 1003-1015.
79. Heilbronner DM, Sussman MD. Early mobilization of adolescent scoliosis patients following Wisconsin interspinous segmental instrumentation as an adjunct to Harrington distraction instrumentation. Preliminary report. Clin Orthop Relat Res. 1988(229):52-58.

80. King R, Trottier T. Variation in care among spinal deformity surgeons: results of a survey of the Shriners hospitals for children. Spine (Phila Pa 1976). 2007;32(13):1444-1449.

81. Tarrant RC, O'Loughlin PF, Lynch S, et al. Timing and predictors of return to short-term functional activity in adolescent idiopathic scoliosis after posterior spinal fusion: a prospective study. Spine (Phila Pa 1976). 2014;39(18):1471-1478.

82. Milbrandt TA, Singhal M, Minter C, et al. A comparison of three methods of pain control for posterior spinal fusions in adolescent idiopathic scoliosis. Spine (Phila Pa 1976). 2009;34(14):1499-1503.

83. Van Boerum DH, Smith JT, Curtin MJ. A comparison of the effects of patient-controlled analgesia with intravenous opioids versus epidural analgesia on recovery after surgery for idiopathic scoliosis. Spine (Phila Pa 1976). 2000;25(18):2355-2357.

84. Klatt JW, Mickelson J, Hung M, Durcan S, Miller C, Smith JT. A randomized prospective evaluation of 3 techniques of postoperative pain management after posterior spinal instrumentation and fusion. Spine (Phila Pa 1976). 2013;38(19):1626-1631.

85. Blanco JS, Perlman SL, Cha HS, Delpizzo K. Multimodal pain management after spinal surgery for adolescent idiopathic scoliosis Orthopedics. 2013;36(2 Suppl):33-35.

86. Blank J, Flynn JM, Bronson W, et al. The use of postoperative subcutaneous closed suction drainage after posterior spinal fusion in adolescents with idiopathic scoliosis. J Spinal Disord Tech. 2003;16(6): $508-512$.
Journal of Multidisciplinary Healthcare

\section{Publish your work in this journal}

The Journal of Multidisciplinary Healthcare is an international, peerreviewed open-access journal that aims to represent and publish research in healthcare areas delivered by practitioners of different disciplines. This includes studies and reviews conducted by multidisciplinary teams as well as research which evaluates the results or conduct of such teams or health

\section{Dovepress}

care processes in general. The journal covers a very wide range of areas and welcomes submissions from practitioners at all levels, from all over the world The manuscript management system is completely online and includes a very quick and fair peer-review system. Visit http://www.dovepress.com/ testimonials.php to read real quotes from published authors. 